\title{
Untersuchungen über das Abklingen der Erregung im Sehorgan nach kurzdauernder Reizung.
}

Von

\author{
Prof. C. Hess (Würzburg).
}

(Mit 1 Textfigur und Tafel I.)

In mehreren Abhandlungen habe ich früher ${ }^{1}$ ) eine Reihe von Erscheinungen bescbrieben, die sich bei geeigneter Versuchsanordnung nach kurzdauernder Reizung des Selıorgans mit farblosem und mit farbigem Lichte beobachten lassen. Da verschiedene Nachuntersucher bisher nicht im Stande gervesen sind, das dort Beschriebene zu sehen, so war mir daran gelegen, eine Methode zu finden, mit der es auch dem in solchen Untersuchungen Ungeübten ohne besondere Hülfsmittel. möglich wäre, sich durch eigene Beobachtung eine Vorstellung von dem Ablaufe des Erregungsvorganges nach kurzdauernder Reizung des Sehorgans zu machen und die Richtigkeit meiner darauf bezüglichen Angaben selbst zu controliren.

Zum leichteren Verständnisse des Folgenden sei kurz angeführt, dass ich nach kurzdanernder Reizung des Sehorgans mit bewegter farbiger Lichtquelle unter den günstigsten Verhältnissen folgende sechs Nachbildphasen unterscheiden konnte: 1. Die primäre Erregung (= Phase 1). 2. Ein kurzes dunkles Intervall (= Phase 2). 3. Ein kurzdauerndes, zum Reizlichte im Allgemeinen gegenfarbiges Nachbild, heller als die Umgebung (- Phase 3). 4. Ein längeres, dunkles Intervall (= Phase 4). 5. Ein längerdauerndes, dem Reizlichte gleichgefärbtes, wenig gesättigtes Nachbild ( $=$ Phase 5), heller als die Umgebung, aber weniger hell als Phase 3. 6. Ein länger dauerndes dunkles Nachbild, das meist nicht unmittelbar nach dem Schwinden der Phase 5 deutlich sichtbar wird, sondern erst eine kurze Zeit später (- Phase 6).

Es ist nun in der That mit einer äusserst einfachen Versuchsanordnung leicht möglich, nicht nur die meisten der von mir bisher

1) Pflüger's Arch. Bd. 49 'S. 190 (1891); Arch. f. Opbthalm. Bd. 40 H. 2 S. 259 (1894); Bd. 44 H. 3 S. 445 (1898); Bd. 51 H. 2 S. 225 (1900); Zeitschr. f. Psychol. u. Physiol. d. Sinnesorgane Bd. 27 (1901). 
mitgetheilten Beobachtungen $\mathrm{zu}$ wiederholen, sondern diese auch nach verschiedenen Richtungen zu erweitern. Es genügt dazu ein einfacher Streif mattweissen Cartons von etwa $10-15 \mathrm{~cm}$ Länge und 3-6 $\mathrm{mm}$ Breite. Man bewegt denselben in einem Abstande von etwa $50 \mathrm{~cm}$ mit mässiger Geschwindigkeit ${ }^{1}$ ) vor dem beobachtenden Auge vorüber, das (bei verdecktem zweiten) auf die Mitte einer gleichmässig dunklen Fläche gerichtet ist.

Ein besonderes Dunkelzimmer ist für diese Versuche nicht nothwendig; einen grossen Theil derselben kann ich an meinem Schreibtische sitzend vornehmen, wenn ich das Auge auf einen gleichmässigen, faltenlosen, dunkelbraunen Vorhang an der gegenüberliegenden Wand richte; es eignet sich jede gleichmässig dunkle Fläche ohne sichtbare Details, z. B. die Oeffnung einer in ein verdunkeltes Zimmer führenden Thüre oder die dunkle Wand eines Dunkelzimmers. Der Geübte braucht kein besonderes Fixirzeichen; für den Ungeübten, der darauf nicht gerne verzichtet, bietet die Methode den Vorzug, dass z. B. auf einer dunkelgrauen Wand ein dunkles oder schwarzes Fixirzeichen angebracht werden kann, da aus früher von mir eingehend besprochenen Gründen die beliebte Anwendung heller bezw. leuchtender Fixirzejchen zu vermeiden ist. Ausserdem kann das foveale Gebiet durch besondere Methoden (s. u.) leicht kenntlich gemacht werden.

Die Lichtstärke des Reizlichtes lässt sich leicht beliebig innerbalb der hier erforderlichen Grenzen variiren, indem man die Neigung des Papierstreifs zu der Lichtquelle oder seinen Abstand von dieser variirt. (Ueber die Untersuchung mit farbigen Lichtern, die gleichfalls mit dieser Methode leicht ausfülrbar ist, siehe unten).

Diese absichtlich möglichst einfach gewählten Versuchsanordnungen lassen sich natürlichi leicht nach verschiedenen Richtungen vervollkommnen: So benutze ich z. B. vielfach, um die zwar in mancher Beziehung vortheilhafte, doch aber oft unbequeme Bewegung des Streifs mit der Hand zu vermeiden, ein langes Pendel mit beliebig regulirbarer (im Allgemeinen sebr geringer) Schwingungsgeschwindigkeit. Der weisse Streif ist nach oben von der Achse des Pendels, in dessen Verlängerung, so angebracht, dass ihn der Beobachter vor einem dunklen Grunde sich bewegen sieht. Durch Vorsetzen passender Gläser bringt man den Streif in den in etwa $50 \mathrm{~cm}$ Abstand verlegten Fernpunkt des Auges, so dass von ihm ein scharfes Bild auf der Netzhant entworfen wird; schon so können, da

1) Als "mässige" Geschwindigkeit bezeichne ich hier eine solche, bei der der bewegte Papierstreif in einer Secunde um etwa $10-25 \mathrm{~cm}$ verschoben wird. 
dann vom Hintergrunde nur ein verwaschenes Bild entworfen wird, etwa störende Details desselben innerhalb gewisser Grenzen unschädlich gemacht werden. Durch Vorsetzen passender röhrenförmiger Blenden lassen sich etwa die Beobachtung störende Nebeneindrücke ausschalten, so dass der Beobachter nur den dunkeln Hintergrund und den sich darüber bewegenden Streifen sieht. Bei anderen Versuchen war der weisse Streif auf einer mit mattschwarzem Tuche bespannten Trommel befestigt, die durch Elektromotor in langsame Umdrehung versetzt wurde.

Die Vorzüge der Untersuchung mit einem streifenförmigen Reizlichte habe ich schon früher ausführlich auseinander gesetzt: Wesentlich sind einmal die Entbehrlichkeit eines Fixirpunktes zur Untersuchung der fovealen Nachbilder, dann die Möglichkeit einer directen Vergleichung dieser letzteren mit den extrafovealen Nachbildtheilen.

Bewegt man einen etwa $6 \mathrm{~mm}$ breiten weissen Streif in der geschilderten Weise am Auge vorüber, so ist unmittelbar hinter ihm Phase 2 als tief dunkles Intervall, dann Phase 3 als heller, zum ersten paralleler Streif sichtbar, der im Allgemeinen ungefähr ebenso breit oder wenig breiter erscheint, als der der primären Erregung entsprechende Streif. Er ist bei Anwendung angenähert farblosen Reizlichtes farblos hell, bei Anwendung farbigen Lichtes in vielen Fällen ungefähr gegenfarbig, z. B. bei Benutzung einer gewöhnlichen Glühlampe zur Beleuchtung des Streifs schön blau oder grünlichblau, dagegen bei Benutzung rothen Reizlichtes häufig schön roth (s. u.).

Die Methode gestattet auch, dem Leser eine genügend genaue Vorstellung von den von mir in der Regel benutzten Lichtstärken zu geben: man sieht die geschilderte Erscheinung mit helladaptirtem Auge auf dunklem (nicht schwarzem) Grunde sehr deutlich schon dann, wenn der schmale Papierstreifen aus einem Abstande von etwa $2-3 \mathrm{~m}$ ron dem Lichte einer gewöhnlichen (hinter dem Kopfe des Beobachters befindlichen) Glüblampe von 25 Kerzen beleuchtet ist.

Der Versuch mit dem langen Papierstreif als Reizlicht ist vor Allem für die Beobachtung des zeitlichen Verlaufes des fovealen Theiles von Phase 3 werthvoll.

Ich habe schon früher gezeigt, dass diese Phase im fovealen Gebiete kurze Zeit später auftritt als im extrafovealen. Es war danach zu vermuthen, dass bei passend gewählter Geschwindigkeit der Bewegung und zweckmässiger Breite des Reizstreifs der foveale Theil des Nachbildstreifs nach hinten ausgebuchtet erseheinen würde. Dies ist (wie ich auf verschiedenen Wegen feststellen konnte) in der That der Fall: sowie der bewegte Papierstreif in unmittelbare Nähe der Fovea gekommen ist, tritt an der entsprechenden 
Stelle des bis dahin geradlinig verlaufenden Nachbildstreifs eine unter geeigneten Umständen recht beträchtliche Ausbuchtung nach hinten auf (vgl. Figur 2). Der Nachibildstreif ist, wie ich gleichfalls schon früher beschrieb, hier etwas schmäler, als auf den extrafovealen Theilen. Sowie das foveale Gebiet überschritten ist, gleicht sich die Ausbucbtung aus und das Nachbild erscheint wieder geradlinig.

Wird die Bewegung des Streifs etwas langsamer ausgeführt, so erscheint oft nur die vordere Grenzlinie des Nachbildes nach hinten ausgebuchtet, die hintere aber geradlinig. Helligkeit und F arbe des fovealen Nachbildtheiles unterscheiden sich bei der angegebenen Versuchsanordnung im Allgemeinen wenig oder gar nicht von jenen der extrafovealen Theile; keinenfalls ist der foveale viel gesättigter oder viel dunkler, als der extrafoveale Theil.

Die Beziehungen dieser ausgebuchteten Nachbildpartie zum fovealen Bezirke lassen sich auf verschiedene Weise genauer untersuchen. Von den von mir benutzten Methoden erwähne ich hier nur einige einfachere, die gleichfalls ohne besondere Uebung eine Controle ermöglichen.

Am einfachsten ist es, den fovealen Bezirk durch extrafoveale Nachbilder kenntlich zu machen: auf einer gleichmässig mattweissen Fläche bringe man etwa zwei schwarze Scheibchen von ca. $1 \mathrm{~cm}$ Durchmesser so an, dass ihre einander zugewendeten Ränder $35 \mathrm{~mm}$ von einander entfernt sind. Fixirt man aus einem Abstande von $1 \mathrm{~m}$ ein feinstes Pünktchen in der Mitte zwischen beiden Scheibchen, so entsprechen die inneren Ränder der letzteren ungefäbr den Grenzen des stäbchenfreien Gebietes nach den üblichen Maassangaben.

Hat man das Pünktchen etwa 20-30 Secunden lang fest fixirt und richtet nun das Auge auf eine dunkle Fläche, so treten die Nachbilder der schwarzen Scheibchen bald als helle, runde Felder auf, die lange genug sichtbar bleiben, um eine Reihe von Nachbildversuchen mit dem bewegten Papierstreif möglich zu machen; durch wiederholtes Auffrischen der Nachbilder ist man leicht im Stande, genügend grosse Beobachtungsreihen anzustellen.

Es zeigt sich nun sehr schön, dass innerhalb des Bezirkes zwischen den beiden hellen Scheibennachbildern der Nachbildstreif deutlich sichtbar ist und die erwähnte Ausbuchtung zeigt. Indem man bei anderen Versuchen den gegenseitigen Abstand der Scheibehen um ein Weniges vergrössert, kann man sich überzeugen, dass für 
den wagerechten Netzhautmeridian die äussersten Grenzen der ausgebuchteten Partie (auf einen Abstand von $1 \mathrm{~m}$ projicirt) vielleicht ein wenig mehr als $35 \mathrm{~mm}$, - für meine Augen etwa 35 bis höchstens $45 \mathrm{~mm}$ - von einander entfernt sind; sie gehen ganz allmählich in den gerade verlaufenden "Theil des Nachbildstreifs über, wodurch eine genauere Massangabe sehr erschwert wird.

Das Wesentliche und Wichtige dieses Versuches ist der Nachweis, dass 1. ein der Phase 3 entsprechendes foveales Nachbild dentlich sichtbar ist, dass 2. dasselbe in den mittelsten Foveatheilen am spätesten auftritt und dass 3. schon innerhalb des stäbchenfreien Bezirkes selbst, von dessen Mittelpunkte nach der Peripherie hin, eine continuirliche und deutlich nachweisbare A enderung in der Reactionsgeschwindigkeit wahrnehmbar ist, derart, dass der foveale Nachbildverlauf allmählich in den für die extrafoveale Netzhant charakteristischen ubergeht. Die Methode gestattet, in der Art der Ausbuchtung der Nachbildlinie die Reactionsgeschwindigkeiten der.verschiedenen Punkte des fovealen Gebietes gewissermaassen graphiscbzu veranschaulichen. Bei bekannter Geschwindigkeit der Bewegung wird es nicht schwer sein, genauere Zeitangaben hierüber zu erhalten.

Bei vielen Beobachtungen benutzte $\mathrm{ich}$, um eine al.Iseitig sichtbare Abgrenzung des fovealen Bezirkes herbeizuführen, statt der Scheibchen einen dunklen Ring von ca. $4 \mathrm{~mm}$ Breite und $35 \mathrm{~mm}$ lichtem Durchmesser auf weissem Grunde. Nach Fixiren des Kreismittelpunktes während 20-30. Secunden aus $1 \mathrm{~m}$ Entfernung sieht man bei Betrachtung einer dunklen Fläche bald einen hellen Ring; dessen innerer Rand ungefähr der Grenze des stäbchenfreien Gebietes entspricht. Bei Bewegung des Papierstreifs über die mittleren Theile des Gesichtsfeldes sieht man den Nachbildstreif sehr deutlich in dem von dem Ringe umschlossenen Gebiete. Mehrere Personen, die in derartigen Untersuchungen wenig oder gar keine Uebung hatter, stellten die fraglichen Beobachtungen ohne Schwierigkeit mit gleichem Ergebnisse an.

Der Geübtere kann den folgenden, etwas schwierigeren Versuch machen: Als Reizlicht benutze man einen nur etwa $1 \mathrm{~cm}$ langen, $4-6 \mathrm{~mm}$ breiten weissen Streif, den man in einem Abstande von $50 \mathrm{~cm}$ vor dem Auge bewegt. (Um den störenden Einfluss der bewegenden 
Hand auszuschalten, genügt es, einen weissen Kartonstreif bis auf eine $1 \mathrm{~cm}$ lange Strecke zu schwärzen und mit dem geschwärzten Theile in der Hand zu halten, oder aber den Papierstreif an einem feinen, mattschwarzen Drahte zu befestigen.)

Man erzeugt sich nun ein kräftiges Nachbild des vorher erwähnten Ringes und bewegt den kurzen Streif so, dass er immer vollständig innerhalb des hellen Nachbildrings gesehen wird. Am zweckmässigsten fand ich, den Streif etwa an den oberen Rand des hellen Ringes zu bringen, nun quer durch dessen Mitte zum unteren Rande zu führen und hier mit der Bewegung einzuhalten. Dann entfernt man ihn rasch aus dem Beobachtungsgebiete und wiederholt die Bewegung nach genügender Pause in gleicher Weise. Bei diesem Versuche werden also überhaupt nur Zapfen gereizt. Trotzden und trotz der in Folge der Nothwendigkeit sehr kleiner rascher Bewegungen besonders ungünstigen Versuchsbedingungen sieht man auch jetzt ein vollkommen deutliches Nachbild (= Phase 3), wenn auch, wie nach den vorhergehenden Schilderungen und meinen früheren Beobachtungen zu erwarten war, schmäler als auf den extrafovealen Netzhauttheilen ${ }^{1}$ ).

Bei anderen Beobachtungen wurden zur Orientirung über den Ort des fovealen Bezirkes die Nachbilder weisser Scheiben oder Ringe von entsprechender Grösse auf dunklem Grunde benutzt, ausserdem die Versuche noch mit verschiedenen anderweitigen Modificationen wiederholt. Die geschilderten Ergebnisse konnte ich so auf mannigfache Weise für meine und andere gesunde Augen bestätigen.

Foveales Fehlen der Phase 3 muss, soweit es nicht auf mangelhafter Beobachtung beruht, als krankhaft bezeichnet werden.

Dass bei hochgradiger Dunkeladaptation und genügend geringer Lichtstärke des Reizlichtes in Folge der langsameren und unvollständigeren Adaptation der fovealen Netzhant (bei Benutzung kurzwelliger Lichter auch in Folge der macularen Absorption) der foveale Nachbildtheil gelegentlich etwas weniger hell erscheinen kann als der extrafoveale, bedarf nach meinen früheren Ausführungen keiner

1) Bewegt man einen solchen Streif derart durch das Gesichtsfeld, dass auch der foveale Bezirk gereizt wird, so kann hier bei passender Geschwindigkeit der Bewegung die Phase 3 schon in Folge ihres späteren Auftretens etwas Discontinuirliches, Sprunghaftes in ihrer Bewegung zeigen. Bei oberflächlicher Beobachtung mag dadurch ein fovealer Ausfall dieser Phase vorgetäuscht werden. 
erneuten Besprechung, ebenso ist selbstverständlich bei so geringer Lichtstärke, bei welcher das Vorbild foveal nicht wahrnehmbar ist, auch das Nachbild foveal nicht sichtbar. Dass aber unter solchen Verhältnissen, wo die Phase 3 dentlich und angenähert gleich lang oder wenig länger wie Phase 1 erscheint, dieses Nachbild auch auf der Fovea zu Stande kommt, ist bei aufmerksamer Beobachtung nach den oben angegebenen Methoden leicht festzustellen.

Der Einfluss der Lichtstärke des Reizlichtes auf die Zeit des Auftretens von Phase 3 ist mit unserer Metbode leicht zu untersuchen. Ich benutzte dazu theils Streifen, die in verschiedenen grauen Tönen hergestellt waren und unmittelbar an einander grenzten, theils solche, die einen allmäligen Uebergang vom hellerem zum dunklerem Grau zeigten. Diese letzteren kann man sich nöthigen Falls selbst herstellen, indem man Graphit oder Tusche in passender Weise auf einen schmalen weissen Cartonstreif aufträgt. Besser ist ihre Herstellung auf photographischem Wege. Jeder Glanz auf dem Streif ist sorgfältig zu vermeiden. (Geringe Unterschiede in seiner Färbung kommen nicht störend in Betracht, solange lediglich die zeitlichen Verhältnisse der Nachbildphasen Gegenstand der Untersuchung sind.)

Bei Benutzung derartiger Reizlichter zeigt sich, dass innerhalb der Grenzen der Sichtbarkeit der Phase 3 diese um so später auftritt, je schwächer das Reizlicht ist: bei einem Streifen mit nach einem Ende hin continuirlich abnehmender Lichtstärke erscheint Phase 3 nicht mehr als ein zum Reizstreifen paralleler Streif, sondern unter spitzem Winkel zu ihm geneigt: am hellen Ende des Reizstreifs ist der Nachbildstreif diesem am nächsten und entfernt sich gegen das dunklere Ende immer weiter von ihm, wobei er gleichzeitig an Breite abnimmt.

Zur Beobachtung der Phasen 4, 5 und 6 (welche von mehreren Nachuntersuchern bekanntlich vollständig übersehen wurden) sind die Methoden mit dem langen Streif weniger geeignet als die folgenden.

Bei einer ersten Gruppe von Versuchen richtete ich mein Augenmerk vorwiegend anf das Verhalten der von mir früher geschilderten dunklen Phase 4, die dem eben besprochenen hellen Nachbilde folgt und eine wesentlich längere Dauer hat als dieses.

Ich hatte bei meinen früheren Untersuchungen mit Reizlichtern auf nahezu lichtlosem Grunde nicht mit Bestimmtheit anzugeben vermocht, ob diese dunkle Strecke dunkler sei, als die seitliche 
Umgebung. Um zu ermitteln, ob das Sehorgan sich während der Dauer dieser Phase im Ruhezustande befinde (was aus verschiedenen Gründen sehr unwahrscheinlich war), benutzte ich die (in der Zeitschr. f. Psych. u. Physiol. d. Sinnesorg. Bd. 27 von mir bescbriebene) Methode der Reizung mittels unterbrochener Lichtlinie. Entsprechend der Stelle der Unterbrechung zeigte sich während der Dauer der Phase 4 ein heller Streif von ansehnlicher Länge. Damit. war der Beweis erbracht, dass die entsprechenden Partien des Sehorgans während der Dauer dieser Phase sich nicht im Zustande der Ruhe befinden, sondern in einem Erregungszustande; welcher bezüglich der Helligkeit dem der. Phase 3 und 5 entsprechenden entgegengesetzt ist.

Danach war zu vermuthen, dass es bei Benutzung eines nicht zu dunklen Grundes möglich sein würde, die Phasen 2 und 4 deutlich dunkler zu sehen als die seitliche Umgebung. Diese Vermuthung wird durch den Versuch bestätigt und damit ein neuer Beweis für -die Richtigkeit jener Auffassung erbracht.

$\mathrm{Zu}$ den früheren Untersuchungen mit bewegter Lichtquelle hat man (so weit nicht der oben geschilderte Lichtstreif in Betracht kam) fast ausschliésslich ein kreisförmig begrenztes Reizlicht benutzt. So lange nur $d^{i} i_{e}$ Beobachtung der auf einander folgenden Phasen des Erregungsvorganges in Betracht kommt, kann dieses Verfahren genügen. Für zahlreiche feinere Beobachtungen ist es aber unzweckmässig, hauptsächlich desshalb, weil hier bei Bewegung der Lichtquelle die einzelnen Netzhautpartien sehr verschieden lange gereizt werden: die mittleren am längsten, die. ihnen beiderseits angrenzenden in dem Maasse kürzer, als sie weiter von der Mitte entfernt sind, die dem Rande des Reizlichtes entsprechenden Theile nur ä usser rst kurż. Es ist begreiflich, dass dadurch für die Beobachtung des Ablaufes der Lrregung die Verhältnisse sehr viel complicirter liegen, als wenn die benutzten Netzhautpartien alle gleichzeitig und gleich lange gereizt werden. Aus diesem Grunde habe ich zu meinen neuen Beobachtungen ausschliesslich rechteckig begrenzte Reizlichter benutzt; die Bewegungsichtung war immer einer Seite des Rechteckes (angenähert) parallel. In der That lassen sich damit verschiedene Erscheinungen wahrnehmen, die mit jenen früheren Methoden nicht sichtbar sind.

Zunächst ist bei Berutzung eines Grundes von passender Hellig. keit mit grösster Deutlichkeit zu sehen, dass die den Phasen 2 und 4 
entsprechenden Stücke der Nachbildstrecke beträchtlich dunkler sind, als die seitliche Umgebung; Pbase 2 ist wieder deutlich dunkler als Phase 4. Ich sehe dies z. B. sehr gut, wenn ich als Grund, über dem die Bewegung erfolgt, die mattschwarze Wand meines Dunkelzimmers benutze, die aus einem. Abstande von ca. $6-8 \mathrm{~m}$ von einer 25 kerzigen Mattglasglühlampe beleuchtet ist. Auch bei Benutzung eines dunkelbraunen, von einer $4 \cdot \mathrm{m}$ entfernten Lampe belichteten Grundes oder einer genügend schwach belichteten weissen Wand ist die Erscheinung sehr deutlich. (Wird der Grund allimählich heller gemacht, so ist bald eine obere Grenze für die Beobachtung durch das Unsichtbarwerden von Phase 3 gegeben).

Sehr auffällig' und schön ist dabei zu sehen, dass 'die seitliche Begrenzung des dunklen Streifs in dem der Phase 4 entsprechenden Theile zunächst eine verhältnissmässig grosse Helligkeit zeigt, die sich allmählich in das gleichmässige Dunkelgrau der weiteren Umgebung verliert:

Diese letztere Erscheinung tritt bei Phase 6 wieder in grosser Deutlichkeit hervor; auch bei Phase 2 ist sie wohl vorhanden, aber wegen der sehr kurzen Dauer derselben nicht ganz so leicht zu sehen. Entsprechend den hellen Phasen 1, 3 und 5 ist die seitliche Begrenzung des Nachbildstreifs sicher nicht heller, für Phase 1. und 5 sogar deutlich dunkler, als der übrige dunkelgraue Grund. (Wohl auch für Phase 3, doch ist hier die Beobachtung durch die kurze Dauer dieser Phase sehr erschwert.)

Auch der früher von mir beschriebene, auf Simultancontrast beruhende helle Nachbildstreif während der Dauer der Phase 4 (s. oben) ist mit der Streifenmethode sehr schön sichtbar zu machen; es genügt dazu, den langen weissen Papierstreif an einer oder mehreren Stellen in ganzer Breite und je auf einer Strecke von etwa $2-5 \mathrm{~mm}$ zu schwärzen.

Bei Bewegung des Streifs sind während der Dauer der Phase 4 an den den schwarzen Partien entsprechenden Stellen des Nachbildes helle Streifen von beträchtlicher Länge sichtbar.

Das foveale Auftreten der Phase 4 lässt sich besonders leicht mit der vorher geschilderten Methode untersuchen, bei welcher das stäbchenfreie Gebiet durch Erzeugung eines seinen Grenzen entsprechenden hellen Nachbildringes erkennbar gemacht ist: Legt man die Bahn eines etwa $1 \mathrm{~cm}$ langen, $4 \mathrm{~mm}$ breiten weissen Streifs, der in $50 \mathrm{~cm}$ Abstand vor dem Auge vorüberbewegt wird, durch 
diesen Ring, so tritt innerhalb desselben die Phase 4 als tief dunkler Streif in hellerer Umgebung auf's Deutlichste hervor.

Endlich ist unsere Methode auch geeignet zur Beobachtung der von mir beschriebenen langdauernden Phasen 5 und 6 .

Es herrschen zum Theile noch ausserordentlich unrichtige Vorstellungen über die Dauer des Erregungsvorganges nach kurzdauernder Reizung des Sehorgans mit Lichtern von der hier in Frage kommenden Stärke: Eine deutlich wahrnehmbare Aenderung des Erregungszustandes nach raschem Vorüberführen eines ca. $5-6 \mathrm{~mm}$ breiten Papierstreifs, der ans $1-2 \mathrm{~m}$ Entfernung von dem Lichte einer 25 kerzigen Mattglasglühlampe bestrahlt wird, ist in dem kurz dunkeladaptirten und vor weiterem Lichteinfalle geschützten Auge of t 12-15 Secunden und n och länger nachweisbar.

In der Regel ist dann der zeitliche Verlauf ungefähr so, dass etwa während der ersten viertel bis halben Secunde Phase 1, 2 und 3 abklingen; etwa am Ende der ersten Secunde entwickelt sich Phase 5 als heller Streifen in dunkler Umgebung und bleibt etwa 4-5 Secunden lang sichtbar. In der 9.-12. Secunde etwa wird dann mehr oder minder deutlich ein dunkler Nachbildstreifen sichtbar, der beiderseits von einer breiten, unbestimmt begrenzten helleren Partie ungeben ist ${ }^{1}$ ). Es ist danach begreiflich, dass man diese drei länger dauernden letzten Phasen übersehen muss, wenn die einzelnen Reizungen in Zwischenpausen von weniger als zwei Secunden anf einander folgen. Setzt man die Lichtstärke des Reizlichtes allmählich herab, indem man etwa den zur Reizung benutzten Papierstreif inmer weiter von der Lichtquelle entfernt, so wird Phase 3 immer weniger deutlich und weiterhin vollständig unsichtbar. Bei Benutzung einer 25 kerzigen Mattglasbirne im Dunkelzimmer ist dies für meine kurze Zeit dunkeladaptirten Augen der Fall, wenn der Streifen mehr als etwa $3^{1 / 2}-4 \mathrm{~m}$ von der Lichtquelle entfernt wird. Von Phase 3 ist dann nichts mehr zu sehen; die langdauernde helle Phase 5 ist jetzt aber noch durch mehrere Secunden sichtbar.

Es ist also ganz unmöglich, die Phase 3 , wie bebauptet wird, mit solehen Lichtstärken des Reiz-

1) Bei sehr oberflächlicher Beobachtung kann es den Anschein haben, als sei mit der Phase 3 die ganze Erregung abgeklungen, als dauere also bei dieser Form der Lrscheinung der ganze Effect der Reizung nur etwa $1 / 2$ Secunde oder noch weniger. 
lichtes überhaupt sichtbar zu machen, für welche der ganze Effect der Reizung nur 1/2 Secunde oder noch weniger dauert.

Mittels des Ringnachbildes überzeugt mau sich leicht, dass auch diese Phasen foveal deutlich sichtbar sind.

Die bisher besprochenen Erscheinungen beziehen sich im Allgemeinen auf farbloses oder angenähert farbloses Reizlicht.

Die Erscheinungen bei farbigem Reizlichte lassen sich mit unserer Methode gleichfalls leicht untersuchen; ich bediente mich unter Anderem der folgenden Versuchsanordnungen:

1. Es wurden zur Reizung $4-6 \mathrm{~mm}$ breite Streifen gesättigt farbigen Papiers benutzt, die, in passendem Abstande vom Tageslichte belenchtet, vor der dunklen Wand des Dunkelzimmers vorübergeführt wurden.

2. Fin weisser Papierstreif wurde von einer gesättigt farbigen Lichtquelle belichtet; als solche diente vielfach eine gewöhnliche Glühlampe, vor der in geeigneter Weise gefärbte Gläser angebracht werden konnten. Sehr zweckmässig fand ich auch die Belichtung des weissen Streifs mittels eines $28 \mathrm{~cm}$ breiten, $56^{\circ} \mathrm{cm}$ hohen, mit gesättigt farbigem Glase versehenen Ausschnittes in dem Laden meines Beobachtungszimmers (ich benutzte dazu die bekannte Einrichtung des Hering 'schen Fensters); die zweckmässigste Lichtstärke konnte leicht durch Aendern des Abstandes vom Fenster erhalten werden.

3. Weiter benutzte ich vielfach zur Färbung des weissen, vom Tageslichte belichteten Streifens farbige Gläser, die ich vor das beobachtende Auge hielt; sehr gute Dienste leisteten mir hierbei die farbigen Glaskeile, die von der Firma Zeiss in den Handel gebracht werden und durch leichtes Verschieben vor dem Auge Lichtstärke und Sättigung innerhalb ziemlich weiter Grenzen zu variiren gestatten.

Als Grund, vor dem der Streif bewegt wurde, diente meist die mattschwarze Wand des Dunkelzimmers, für gewisse Beobachtungen eine genügend schwach mit farblosem oder farbigem Lichte erhellte weisse Wand.

Für diese Versuche mit farbiger Lichtquelle (die hier nur ganz kurz erwähnt werden sollen), ist zu berücksichtigen, dass bei ibnen das Auge mehr oder weniger stark für farbiges Licht ermüdet ist, und dass auch der Grund, über den der Streif bewegt wird, nicht 
ganz farblos ist. Ein Gleiches gilt, wenn auch in weniger hohem Maasse, für die Untersuchung bei Tageslicht, das ja bekanntlich fast immer mehr oder weniger ausgesprochen gefärbt ist. Diese Umstände, insbesondere die chromatische Adaptation, können auf die Färbung der verschiedenen Nachbildphasen von wesentlichem Einflusse sein.

Bei meinen frúheren Untersuchungen war zur Reizung ein farbiger Fleck oder Streif auf angenähert lichitlosem Grunde im Dunkelzimmer benutzt worden, das Auge also angenähert chromatisch neutral gestimmt. Für diese Versuchsbedingungen fand ich in der Regel Phase 3 zum Reizlichte gegenfarbig, Phase 5 dagegen in gleicher Farbe wie letzteres, sofern dieses genügend gesättigt war; bei ungesättigter Färbung des Reizlichtes erschien Phase 5 angenẗhert farblos. In den dunklen Phasen 2, 4 und 6 konnte ich eine Färbung nicht nachweisen; dagegen zeigte das während der Dauer der Phasen 2 und 4 nach der oben gesclijderten Methode hervorgerufene, auf Simultancontrast beruhende Nachbild für Phase 2 eine mit dem Reizlichte übereinstimmende Färbung, für Phase 4 eine zu ihm gegenfarbige.

Dass diese Regeln aber keineswegs allgemein gelten, habe ich schon früber gezeigt und insbesondere hervorgehoben, dass bei Benutzung gesättigt rothen Reizlichtes Phase 3 gleichfalls roth gesehen werden kann.

Auch diese Thatsachen lassen sich mit unserer einfachen Methode leicht controliren. Benutzt man zur Reizung etwa einen schmalen Streifen leicht gelblich rothen Papiers, so erseheint Phase 3 in einem leicht bläulich rothen Tone. Dass sie zeitlich wirklich mit der Phase 3 bei andersfarbigem Reizlichte übereinstimmt, zeigt unter Anderem der folgende Versuch: Man benutze zur Reizung einen schmalen Streif, der etwa zur einen Hälfte aus hellgrauem, zur anderen aus dem rothen Papiere besteht und der von angenähert farblosem Lichte beleuchtet wird. Phase 3 erscheint dann entsprechend der farblosen Hälfte hellgrau, entsprechend der rothen roth; der rothe Nachbildstreif ist etwas schmäler als der hellgraue und seine vordere Grenzlinie eine Spur weiter vom Reizstreif entfernt als die des farblosen. Dies entspricht der Thatsache, dass die weisse Valenz des rothen Reizlichtes verhältnissmässig sehr klein ist gegenüber jener des hellgrauen: Bei stark herabgesetzter Beleuchtung erscheint die graue Hälfte des zur Reizung benutzten 
Streifs sehr hell, fast weiss, die rothe dagegen dunkelgrau. Nimmt man statt des hellorauen einen durkelgrauen Reizstreif von genügend geringer Helligkeit, so treten die Nachbildstreifen für die dunkelgraue und die rothe Streifenhälfte gleichzeitig auf und unterscheiden sich nur durch die Färbung von einander (s. Fig. 3).

Eine dem Reizlichte ähnlich gefärbte Phase 3 kann man übrigens auch bei anderem als rothem Reizlichte beobachten: In einem von einer 25 kerzigen Mattglasglühlampe beleuchteten Dunkelzimmer erhielt ich von einem gesättigt blauen Papierstreif eine dentlich blaue Phase 3; dagegen war bei Bewegung eines durch ein sehr gesättigt blaues Glas gesehenen blauen Papierstreifs Phase 3 gelb. Die Erklärung für diese Verschiedenheit ist (neben der nicht ganz gleichen Färbung des Reizlichtes) wesentlich in der oben erwähnten Verschiedenheit der chromatischen Stimmung des Sehorgans in beiden Fällen zu suchen. Die einschlägigen Verbältnisse werden bei anderer Gelegenheit eingehender zu erörtern sein.

Dass für Farbenblinde bei Beurtheilung dieser farbigen Nachbilder sich eine gewisse Vorsicht empfiehlt, könnte überflüssig erscheinen zu erwähnen, wenn nicht kürzlich ein Rothgrünblinder die Existenz eben dieses rothen Nachbildes eines rothen Reizlichtes ganz ernsthaft für das gesunde Auge in Abrede gestellt hätte.

Auch den viel erörterten Einfluss hochgradiger Dunkeladaptation auf die Sichtbarkeit von Phase 3 untersuchte ich abermals mit der neuen Methode.

Mit eivem Streif von passend abgestufter Helligkeit konnte ich nachweisen, dass auch nach mehrstündigem vollständigem Lichtabschlusse die Phase 3 nicht wegfällt, sondern im Wesentlichen in gleicher Weise auftritt wie im helladaptirten Auge, sofern der gesteigerten Erregbarkeit des Sehorgans Rechnung getragen wird (was man bei diesbezüglichen Beobachtungen oft ausser Acht gelassen bat). Auch wenn ich nach mehrstündiger Nachtruhe im völlig verdunkelten Raume erwache und nun mit dem nur während der Bewegung belichteten Streif in passendem Abstande von der Lichtquelle experimentire, ist die Phase 3 deutlich sichtbar (bei Anwendung farbigen Reizlichtes natürlich viel weniger gesättigt als für das helladaptirte Auge). Der adaptative Ausfall der Phase 3, der bekanntlich als besonders wichtige neue Thatsache beschrieben worden ist, muss somit auf mangelhafte Beobachtung zurückgeführt werden, sofern er nicht etwa in krankhaften Veränderungen seinen Grund hat.

Nachdem mit den geschilderten Methoden der Nachweis gelungen ist, dass die Nachbildphasen 2, 4 und 6 deutlich dunkler erscheinen 
als der dunkelgraue Grund, auf dem sie gesehen werden, ist vielleicht der Versuch einer graphischen Darstellung des Nachbildverlaufes

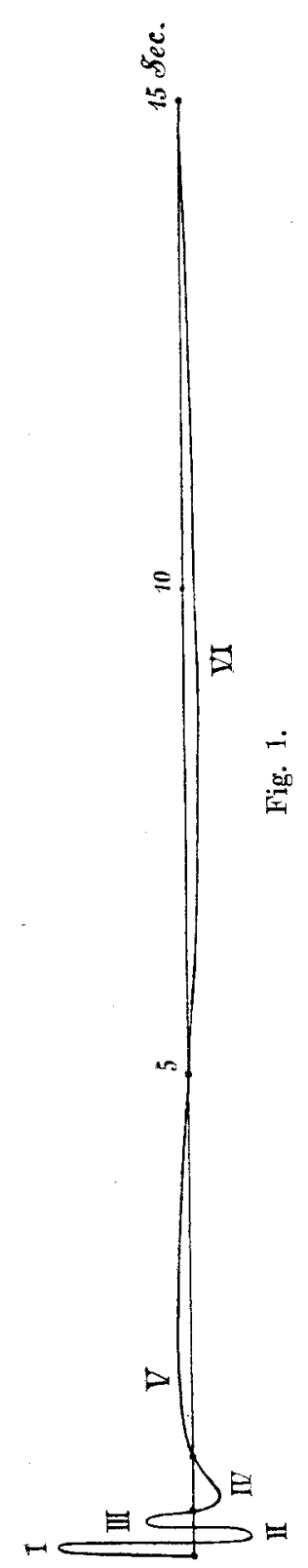
nach kurzdauernder Reizung mit farblosem Lichte gestattet. Freilich kann eine solehe Wiedergabe nur sehr bedingten Werth haben; denn sie gilt immer nur für die jeweiligen Voraussetzungen hinsichtlich der Lichtstärke und Grösse des Reizlichtes, der Dauer der Reizung und der Stimmung des Sehorgans. Aber selbst dann wird ein solches Diagramm nicht viel mehr sein können als eine ziemlich grob schematische Wiedergabe des unter den gegebenen Verhältnissen eindringlichsten Theiles der Erscheinungen; mit Verfeinerung der Beobachtungsmethoden werden sich diese Erscheinungen wohl noch weiter auflösen und dementsprechend zutreffender wiedergeben lassen, als es heute möglich ist. Trotz aller dieser Be$\rightarrow$ denken scheint es mir nicht überflüssig, das besonders Characteristische und Eindringliche im Nachbildverlaufe durch ein solches Schema zu veranschaulichen. Man nahm früher bekanntlich an, dass nach Aufhören des Reizes die Erregungsstärke anfangs sehr schnell, dann immer langsamer heruntersinke und hat dies durch eine Curve ausgedrückt, die der Abscissenachse ihre Convexität zukehrt. An Stelle dieser Auffassung (deren Unhaltbarkeit bereits in meiner ersten hierauf bezüglichen Abhandlung nachgewiesen wurde) ergibt sich jetzt Folgendes: Trägt man in einem Coordinatensystem auf der Abscissenachse die Zeiten derart ein, dass je $1 \mathrm{~cm}$ einer Secunde entspricht, als Ordinaten die Helligkeiten, in welchen etwa die verschiedenen Phasen gesehen werden, verglichen mit der Helligkeit des Grundes, so erhält man für die obigen Versuchsbedingungen ungefähr ein Diagramm von nebenstehendem Typus (Fig. 1). Es bringt einigermaassen den zeitlichen Verlauf der verschiedenen Phasen sowie die 
Thatsachen zum Ausdrucke, dass Pbase 1,3 und 5 heller als der Grund erscheinen, Phase 3 beträchtlich weniger hell als Phase 1 und viel heller als Phase 5; ferner, dass Phase 2, 4 und 6 deutlich dunkler erscheinen als der Grund, Phase 2 dunkler als Phase 4. Dass bezüglich der relativen Höhe der Ordinaten der einzelnen Curventheile es sich selbstverständlich nur um ganz ungefähre, auf irgend welche Genauigkeit nicht Anspruch machende Schätzung handeln kann, braucht nicht betont zu werden. Sieht man davon ab, so gibt das Diagramm lediglich die Thatsachen wieder, besteht also unabhägig von jeder Theorie zu Recht.

Wollte man etwa die zeitlichen Verhältnisse viel genauer verzeichnen, so müsste für die Darstellung des fovealen Abklingens der zweite Curvengipfel nach dem oben Gesagten ein wenig weiter vom ersten entfernt und etwas kürzer sein, als für die des extrafovealen. Der Typus des Abklingens aber, insbesondere das Vorhandensein von drei hellen und drei dunklen Phasen, ist für den nur Zapfen enthaltenden Bezirk ganz der gleiche wie für den auch Stäbchen haltenden.

(Hinsichtlich der Erklärung der Farbe von Phase 3 und 5 im Allgemeinen, und insbesondere der rothen Färbung von Phase 3 bei rothem Reizlichte aus der Theorie der Gegenfarben verweise ich auf meine früheren Abhandlungen.)

Es ist vielleicht nicht überflüssig, zu betonen, dass ich die hier geschilderten Erscheinungen nicht etwa lediglich mit den hier besprochenen Methoden untersucht habe: Das einschlägige Gebiet wurde vielmehr auch neuerdings wieder von mir mit verschiedenen neuen Vorrichtungen untersucht, bei welchen Lichtstärke und Sättigung der Reizlichter sowie ihre Bewegungsgesehwindigkeit innerhalb weiter Grenzen beliebig variirt werden konnten und insbesondere auch Vergleichungen zwischen verschieden lichtstarken und verschieden farbigen Reizlichtern in beträchtlichem Umfange möglich waren; ich behalte mir vor, hierüber bei anderer Gelegenheit eingehender zu berichten. Mit den vorliegenden Zeilen verfolgte ich wesentlich den Zweck, zu zeigen, dass auch der in solchen Untersuchungen Ungeübte sich von der Richtigkeit der mitgetheilten Beobachtungen ohne besondere Hülfsmittel - lediglich mit einem Papierstreif leicht überzeugen kann.

Dass die Dreifasertheorie sowoll in ihrer älteren Fassung wie in ihren jüngeren Modificationen vollkommen ausser Stande ist, die 
16 C. Hess: Untersuch. üb. d. Abklingen d. Erregung im Sehorgan etc.

hier zusammengestellten Thatsachen zu erklären, bedarf nach dem Gesagten wohl nicht mehr eines besonderen Nachweises.

\section{Tafe lerklär nng.}

Auf Tafel I habe ich den Versuch gemacht, einen Theil der im Vorstehender geschilderten Erscheinungen wiederzugeben. Wenn ich auch wohl weiss, dass es kaum möglich ist, diese flüchtigen Phänomene ganz naturgetreu abzubilden, so schien uns doch eine Wiedergabe des charakteristischen Theiles der Erscheinungen zur Erleichterung des Verständnisses und der Nachuntersuchung wünschenswerth, um so mehr, als einige frühere Abbildungen vielfach unrichtige Vorstellungen von den Thatsachen geben. Ich habe den Künstler, Herrn W. Freytag, nur auf die hauptsächlichsten Punkte aufmerksam gemacht und ihn im Uebrigen die Zeichnung nach seinen eigenen Beobachtungen anfertigen lassen. (Auf gewisse Besonderheiten, welche für die uns hier zunächst interessirenden Fragen weniger in Betracht kommen, ist nicht Rücksicht genommen worden, so z. B. auf die Tonänderungen, die man bei geeigneter Versucbsanordnung an den einzelnen Theilen der Phase 1 selbst wabrnehmen kann, und auf eigenthümliche Formänderungen, welche Phase 3 unter Umständen zeigt.)

Fig. 1 gibt eine Uebersicht über die sechs Nachbildphasen nach Reizung des Sehorgans mit dem vor einer dunkeln Fläche mässig schnell bewegten kurzen weissen Papierstreif, der aus etwa 1 bis $2 \mathrm{~m}$ Entfernung von dem Lichte einer 25 kerzigen Mattglasglüblampe beleuchtet ist. Die Phasen 5 u. 6 sind (mit Rücksicht auf den verfügbaren Raum) im Verhältniss zu den ersten Phasen viel zu kurz gezeichnet. Bei richtigem „Verhältnisse müsste jede von beiden mehr als fünf Mal so lang gezeichnet sein als hier (vgl. S. 10).

Fig. 2 zeigt das Verhalten von Phase 3 auf der Fovea bei Bewegung eines langen, schmalen, von einer Mattglasglühlampe beleuchteten Papierstreifs über den fovealen Theil des Gesichtsfeldes: der foveale Abschnitt des Nachbildstreifs. ist nach hinten ausgebuchtet und schmäler als der extrafoveale (vgl. S. 4). (Es sind nur die vier ersten Phasen, und von diesen nur die mittleren $A b$ schnitte abgebildet.)

Fig. 3 zeigt das Verhalten von Phase 3 bei Reizung mit einem schmalen, zur einen Hälfte rothen, zur anderen Hälfte grauen, von Tageslicht beleuchteten. Papierstreif (vgl. S. 12). 
Fig. 1.

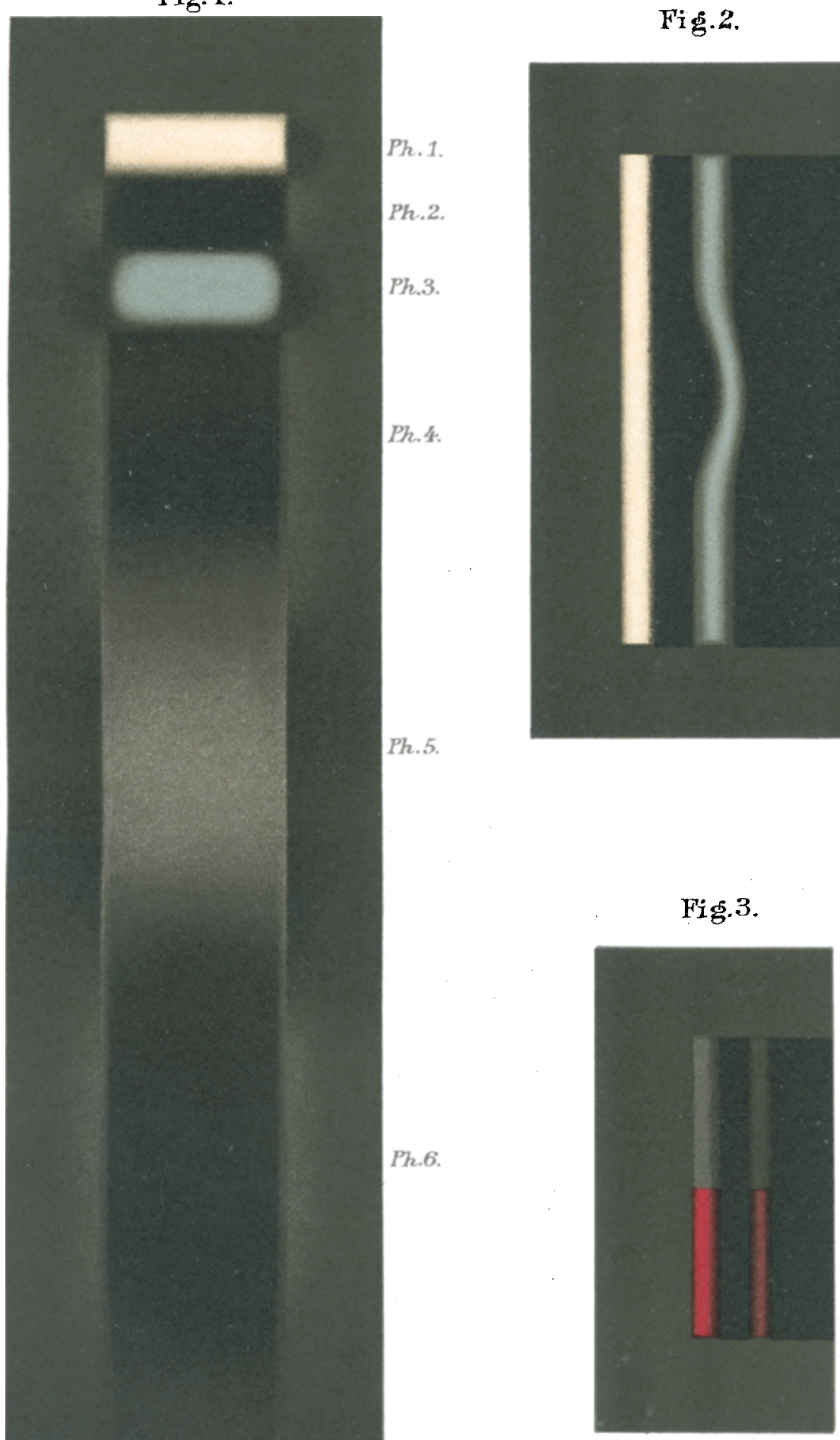

